\title{
Graph Based Filtering and Matching for Symbol Recognition
}

\author{
Vaishali Pawar, Mukesh Zaveri \\ Department of Computer Engineering, SVNIT, Surat, India \\ Email: vaishali_s_pawar@yahoo.com,mazaveri@gmail.com
}

How to cite this paper: Pawar, V. and Zaveri, M. (2018) Graph Based Filtering and Matching for Symbol Recognition. Journal of Signal and Information Processing, 9, 167-191.

https://doi.org/10.4236/jsip.2018.93010

Received: February 22, 2018

Accepted: August 6, 2018

Published: August 9, 2018

Copyright $\odot 2018$ by authors and Scientific Research Publishing Inc. This work is licensed under the Creative Commons Attribution International License (CC BY 4.0).

http://creativecommons.org/licenses/by/4.0/

\section{c) (i) Open Access}

\begin{abstract}
Pattern recognition is a task of searching particular patterns or features in the given input. The data mining, computer networks, genetic engineering, chemical structure analysis, web services etc. are few rapidly growing applications where pattern recognition has been used. Graphs are very powerful model applied in various areas of computer science and engineering. This paper proposes a graph based algorithm for performing the graphical symbol recognition. In the proposed approach, a graph based filtering prior to the matching is performed which significantly reduces the computational complexity. The proposed algorithm is evaluated using a large number of input drawings and the simulation results show that the proposed algorithm outperforms the existing algorithms.
\end{abstract}

\section{Keywords}

Graphs, Pattern Recognition, Information Retrieval, Graph Matching, Data Mining

\section{Introduction}

In pattern matching application, a retrieval of input patterns from a database of model patterns is the most important issue. In graph based recognition techniques the model symbols and the input images are also represented using the primitive graph or by a set of sub graphs. These sub graphs may be considered as a pattern for symbol recognition. In this context, the graph based symbol recognition is the pattern matching task. Graph is always a versatile tool for representing and modeling the patterns. Graph can contain huge amount of data due to its representational power. So they are preferred to represent objects with a huge number of attributes. Finding object similarity is an important issue in many applications such as pattern recognition, information retrieval and data 
mining etc. Design strategy of any pattern recognition or matching algorithm depends on the representation of the object under consideration and attributes of the object. If graphs are used for object representation, then the problem of determining the similarity of objects becomes the problem of graph matching.

The general form of graph matching problem includes the graph and subgraph matching algorithms. An inevitable disadvantage of most of graph matching techniques is that they all suffer from high computational complexities [1] [2] [3] [4]. The problem becomes more complex if the database is large in size. In this context, it is essential to reduce computational complexity using different techniques like dimension reduction, pre-processing of the data, and transformation of data to feature space etc. One of the preprocessing techniques for compact representation of data is filtering which is applied prior to the matching. As the data is represented with a large set of attributes using the graph, there is a need to have an efficient graph based filtering approach to handle this task. The main purpose of having graph database filtering is to reduce the number of database candidates to be matched against the input. In this paper, we present a novel shape based graph database filtering algorithm for optimizing the symbol recognition which is robust and efficient in terms of performance. The overall process of graph matching is to find a good mapping between the vertices and edges of two graphs that satisfy some constraints [5] [6]. In a broad sense, there are two classes of graph matching: exact graph matching and inexact graph matching. In the exact matching [7] [8], a strict bijective mapping is expected between the two graphs to be matched. Graph isomorphism [7] [8] and sub graph isomorphism [7] [8] can be considered as the subclasses of exact graph matching. In the inexact matching [5], the bijective condition is considerably relaxed. The inexact graph matching algorithms are more flexible to accept errors and distortions of the matching object graphs. Therefore, inexact graph matching is also known as error-tolerant graph matching [5] [7] [8]. The rest of the paper is organized as follows. Section 2 describes the literature survey of different graph matching algorithms. Section 3 introduces the proposed the graph based filtering technique. The simulation results are described in Section 4. It is followed by the conclusion and future work.

\section{Literature Survey}

In all classes of graph matching, subgraph isomorphism is a NP-complete problem [9] [10]. But it is still an open research area to decide whether the graph isomorphism is a P or NP-complete problem [9]. The time complexity for finding the isomorphism between trees or bounded valence graphs [11], planar graphs [12], is of polynomial order [6] [13]. The time taken by a brute force approach for graph matching increases exponentially with the size of the input graph [14]. So graph matching algorithms are constrained to contain small number of edges and vertices [1]. One approach to reduce graph matching complexity is to restrict the graph sizes by adopting application specific graph matching [14]. Another attractive solution is to use very accurate searching 
process and pruning unprofitable paths in the search space [1] [6] itself. In graph pruning approach there is no restriction on the size and structure of the graphs to be matched. Some algorithms make use of database clustering [15] and filtering [16] techniques to narrow down the search space of graph matching. Our algorithm is based on graph symbol database hierarchical filtering which is applied prior to the graph matching process. A hierarchically structured approach to organize large structural databases has been presented in [17]. The patterns are modeled in the form of random parametric structural descriptions (RPSDs). Objects in image are modeled as parametric structural descriptions (PSDs). The organization process is controlled by pairwise dissimilarity values between RPSDs. The node pointer lists are computed offline during model base organization [17]. During recognition, the only exponential matching process involved is between the scene PSD and the RPSD at the root of the organized tree. Using the organized hierarchy along with the node pointer lists, the task is reduced to tree matching which linearly tests at the subsequent levels of the tree [17]. Other approaches like hierarchical [18] and hashed [19] databases have been presented for an efficient graph database organization. In most of the algorithms the overall process of graph matching is subdivided into preprocessing part and the actual matching part. The preprocessing part reorganizes the graph database which reduces the graph searching and matching.

In [4] [20] a decomposition approach for model graph has been suggested. The whole model graph data is modeled as network of decomposed graph. [4] uses decomposition approach for preprocessing of the model symbols. Due to the offline preprocessing and filtering of the database, graph matching time becomes sublinearly dependent on the database size [4]. In the last few years, few attempts have been made to combine the best of the graph and the vector domains in order to get the advantages of both techniques. Another approach for graph database reduction using feature vector has been suggested in [21]. In [21], a dissimilarity representation for graphs is used to explicitly transform graphs into $\mathrm{n}$-dimensional feature vectors. This embedding fills the representational gap between the high capacity of graphs and the large amount of classification algorithms available for feature vectors [15] [21]. The basic idea used in this approach is to catch the dissimilarities of predefined model graphs as features. [16] [22] focuses on the importance of graph filtering. In these approaches, the feature vectors are extracted from the graph database and the decision tree is built using these feature vectors. It is followed by graph based matching. The decision tree helps in eliminating the number of candidates from comparison. The approach proposed in [16] [22] is mainly dealing with random graphs generated. The disadvantage of the decision tree is that it is computationally very expensive to built the decision tree and traversing it with larger complex database. In brief, the type of the features and the representation of an object in the database for a given application also play very important roles. The main focus of our work is to deal with real life applications like document image analysis using the graph. For example, the symbol recognition and input drawing analy- 
sis which are very important applications for architectural and historical perspective. For these applications, the first and most important task is to extract the features as these are not regular or structured objects. Second, using these features how to represent the complete object, i.e., input drawing image or symbol for recognition, using the graph so that efficient graph based matching algorithm can be implemented.

\section{Problem Statement}

Although several statistical techniques have been proposed for symbol recognition [23] [24], graph based structural methods always play their prime role. The selection of graph based pattern matching strategy is always subjective to type of pattern. The selection of appropriate type of graph for specific applications with appropriate graph matching algorithm is the key. The symbols appearing in the graphical symbol recognition are not specific and regular in nature. Especially archived documents, historical documents or images are in degraded form. The analysis, content retrieval, restoration, digitization of such data is a challenge. These images are distorted or noisy. Many document images are not clear. These symbols have enormous attributes. It is always a challenge to achieve a graph based solution for any pattern matching application which can perform or work in the presence of distortion and noise. Even if using graphs is the wise option, the size of graph databases is quite large. Storing symbols in database and selecting features for graph matching are key factors. There is no generalised, the best suitable policy available for all the real life pattern matching applications. It is important to have a mechanism which will reduce heavy time complexity of graph matching techniques. Graph based filtering is a process of pruning object database to optimize graph matching. Applying the graph based filtering algorithm prior to graph matching reduces the size of database. As for the sake of convenience, we consider architectural symbol recognition. The database contains model symbols that are used as symbols in architectural drawings. Both the database and input image are modelled using region adjacency graph. When an input architectural plan image is given to identify symbols from the model database, it is an efficient approach to perform filtering and then matching. In this context, it is required to have filtering algorithm, specifically the graph based filtering, as we model input using graph.

\section{Problem Formulation}

The basic concept behind our algorithm is to save expensive graph matching operations by performing prior graph database filtering. The main purpose of our work is to propose a graph based novel algorithm for graphical symbol recognition. The major application of our proposed work is to reconstruct or restore historical or archeological survey plans or drawing which are available as input images which may be distorted or corrupted due to aging effect. Another application is to automate symbol recognition of online web application like 
copyright protection for trademark symbol, product review or sign language interpretation. In brief, we want to propose an algorithm for model symbol recognition where the model symbol may be regular or irregular shape like window, shelf, bed or any architectural symbol etc. These model symbols can be represented using primitive graphic structures like triangle, rectangle or irregular shape geometry etc. It allows one to represent original object in a better way to ease the process of pattern extraction and pattern matching. Here, only assumption is that any regular or irregular shape may be represented by the graph.

In the context of above discussion, we exploit the graph to represent any model symbol in our input image. Throughout the work, the terms, the primitive graphic symbol or model symbol, are used interchangeably with the meaning that it forms a single closed region or an entity or a set of closed regions and a set of entities which can be used as a model graph when the given graphical symbol object or input drawing is decomposed and represented by the graph. In short, from a given object the features or attributes are extracted and these features or attributes can be described using the graph. For example, a simple triangle has three sides and three angles which form an entity or a closed region with sides and angles as features or attributes of a given object. This triangle becomes a graph with a single vertex, representing a closed region with angles, area, sides as attributes of the vertex.

In our proposed algorithm, we use such graphical symbol objects or input drawings as an input which are decomposed into a set of closed regions or entities as described above and stored into the database. For each entity, we extract the features like area of the shape, angles if any, sides, centroid etc. which are nothing but attributes of an entity or primitive graph model symbol. The database with these decomposed objects is called as a graph database. Another important feature that we extract from the input is region adjacency graph (RAG) which provides neighborhood information for a set of closed regions or entities that how they are interconnected with each other. This is also stored as an attribute of a vertex along with other attributes. The RAG formation is the process of establishing a relationship between regions with the help of extracted features.

Our main task is to use these features and attributes to filter out the graph database to reduce to number of model graph comparisons with a test input. To perform this, we index our database using the features and attributes of a vertex. Here, the meaning of indexing is to reorganize the graph database by grouping of model symbols having similar entities or closed regions as a part of given model symbol. Then, searching is performed using this indexed database which is nothing but filtering of the database. This process is explained as shown below in Figure 1. For example, given a graphical symbol or input drawing image having two objects, say, $S_{1}$ which is a staircase and $S_{2}$ is a shelf. $S_{1}$ is decomposed into a set of rectangles and $S_{2}$ is decomposed into four triangles as a set of closed regions or a set of entities, respectively. These model symbols are preprocessed and represented as region adjacency graphs. In RAG, each closed region is a 


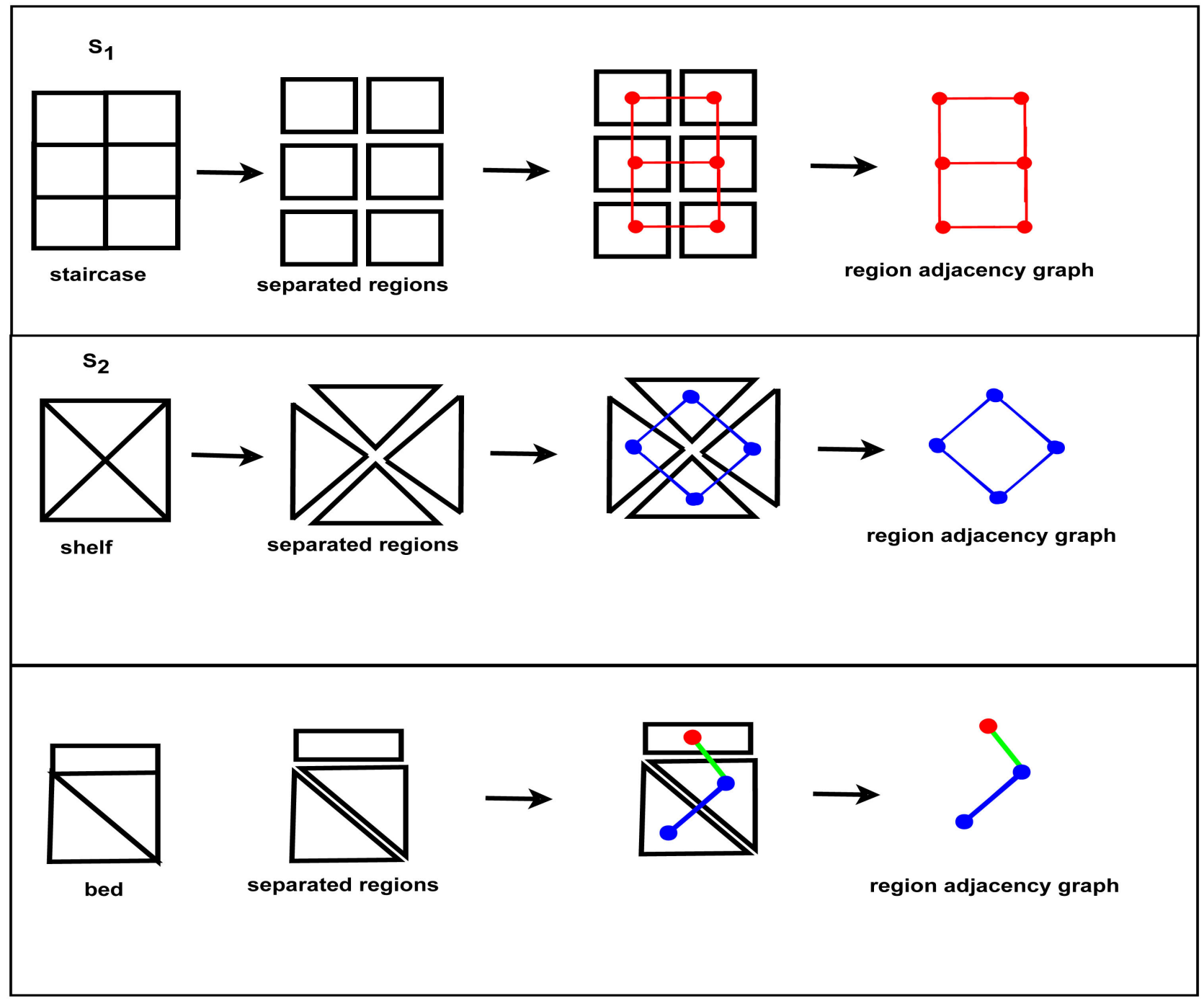

Figure 1. Symbol representation.

vertex. The adjacent regions' attributes are represented by the edge between them. As depicted in Figure 1, symbol $S_{1}$ is a model staircase, $S_{2}$ is a shelf and $S_{\mathrm{n}}$ is a bed. The vertex of RAG represents a closed region in the symbol. The edge between two vertices in a RAG represents the neighbourhood attributes of the two adjacent regions in the symbol. The staircase contains all same regions which are rectangular in nature. The RAG of staircase is shown in red colour in a symbolic way. It indicates that all the vertices are of same type. So all the vertices are in red colour. The adjacency of the vertices is shown by the edges. As all the vertices have similar attributes, all the edges are also carrying similar edge properties; hence, all edges are red in color.

The symbol $S_{2}$ is a shelf, which contains four similar closed regions which are triangular in nature. The RAG of shelf in Figure 1 contains four blue vertices and four blue edges, which indicate that vertices properties and edges properties have similarity. The third symbol $S_{n}$ shown in Figure 1 is a bed. It comprises of two triangular and one rectangular region. The red vertex represents attributes 
of rectangular region. The blue vertices represent triangular regions. The blue edge represents neighbourhood of two blue vertices. The green edge represents neighbourhood of one red and one blue neighbouring vertex.

After representing the model symbols as RAG, the objects are indexed in the database based on the shapes. We propose two variants, linear filtering and the hierarchical filtering. The linear filter filters the symbols in a linear manner, based on the number of shapes. If the model symbols contain exclusive shapes, linear filter performs well. If the input image contains almost all symbols, linear filter performs as good as no filtering of the database. The hierarchical filter arranges the symbol indices based on hierarchy of shapes. It performs well even if the input image is a good mix of all types of shapes.

For example, $S_{1}$ and $S_{2}$ are indexed using rectangle and triangle which allows one to do further filtering. If a given input object is decomposed such that one of the closed region is a rectangle then directly it searches to the subgroup of objects in the database which is indexed using rectangle which avoids a linear filtering or comparison with all objects stored in the database. If a given object is having different types of closed regions then it is indexed using each type of closed region which it consists of. The advantage of such representation is that for searching a given object; the algorithm explores only the subgroup of objects for which an index is matching, which is nothing but filtering out the large number of objects from database being compared. The filtering algorithms are elaborated in the next section. It also is important to note that the features or attributes can be of great help to filter the database. Given a graph database, the salient features are extracted by a feature extraction procedure. The procedure itself should be fast and efficient. The selection of features should be based on the nature of application. Once the features of the graph are extracted, then the database is filtered and only the selective graphs are matched against the input graph. So the matching of an input graph against all prototypes in the database is avoided. If the filtering process is fast enough, the graph matching algorithm saves considerable number of comparisons.

Moreover, the feature extraction must be efficient and fast. The selection of features to extract and to match, affect the filtering and graph matching procedure. The features should have the ability to discriminate between as many graphs in the database. If the given feature can distinguish more graphs, less graph candidates remain after filtering and potentially it achieves efficiency for the expensive matching task. For example, consider feature, constituent shapes of the model symbol. Consider two symbols $S_{1}$ and $S_{2}$ as shown in Figure 1. The symbol $S_{1}$ contains only rectangles while $S_{2}$ contains only triangles. If the query image contains only rectangle, it has to be compared with $S_{1}$. If the query image contains only triangle, it has to be compared with $S_{2}$. If the query image contains both, rectangle and triangle, it has to be compared with both, $S_{1}$ and $S_{2}$. Hence, the selection of feature shape, can play significant role in database filtering and matching. For filtering, graph, sub graph or error tolerant sub graph isomor- 
phism [2] [3] [25] can be used. Using above formulation, the proposed graph based filtering algorithm is described in the following section.

\section{A Novel Algorithm for Graph Filtering and Matching}

For graph based symbol recognition the query image is given as input to the algorithm. The input drawing may consists of many graphical symbols. The feature extraction is performed. The closed regions are extracted. The input image is represented as a RAG. The graph based symbol database contains the dictionary of all symbols used in the application. These symbols are called database model symbols. The graph based filtering and matching is performed to identify the occurrence of model symbols in the input image. Figure 2 explains the concept of graph based filtering and matching. The important thing to note is, filtering is a process carried out during graph matching. While matching the input image graph $G_{1}$ and the model symbol graph $G_{M}$ graph based filtering is performed. The filtering algorithm selects few promising symbols from the model database and filters out the non-promising ones. The graph matching algorithm matches the input graph with the selected promising model symbol graphs.

In our approach, we propose two types of the filtering, namely, graph based linear and hierarchical filtering for graphical symbol recognition. The overall symbol recognition is achieved in three steps as preprocessing, graph filtering followed by the graph matching. The filtering algorithm filters the model symbol database. If the model symbol $G_{M}$ is likely to be similar with the symbols of the input image, it selects $G_{M}$ as promising symbol else it is non-promising. We perform a shape based graph database filtering of the database. For filtering we consider two approaches as 1 . Shape based graph database linear filtering and 2. Shape based graph database hierarchical filtering.

After the graph database filtering, the number of model symbols to be matched with the input graph reduces considerably, which reduces graph matching time. We use region adjacency graph (RAG) based string growing algorithm [7] for the graph matching. In brief, the overall symbol recognition is achieved in three steps 1) preprocessing and feature extraction, 2) the graph database filtering and 3) the graph matching algorithm. In our implementation, we

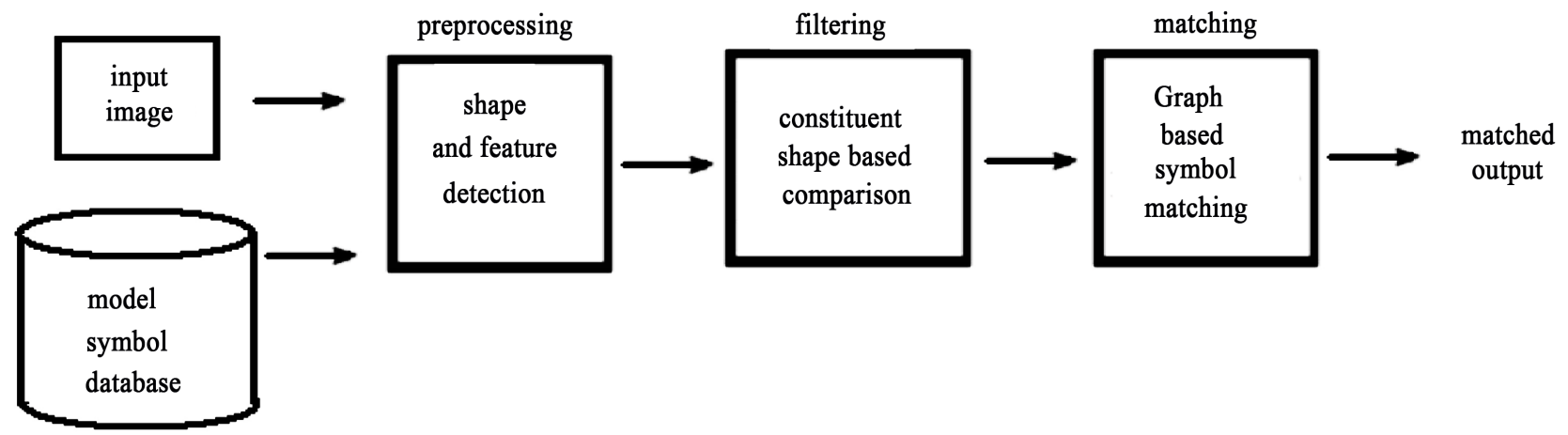

Figure 2. Graph based database filtering and matching. 
use RAG based string growing algorithm [7] for the graph matching. We compare our hierarchical graph database filtering with a standard linear graph database filtering approach to show efficacy of our proposed algorithm. These steps are described in detail as below.

\subsection{Preprocessing and Feature Extraction}

In this step, the input image and the model symbols of the database are preprocessed. It performs primitive operations like thinning, bridging and identification of junctions and end points, region separation etc. for the model symbols and input image. It extracts features like edges, corners, junctions, end-points, closed regions in the image. Each closed region is treated as a basic constituting entity in the symbol or input image. The structural features like area of region, centroid and shape of region etc. are extracted for each region. After region wise feature extraction, the symbols and input image is represented as a RAG. Each closed region is represented as a vertex of the graph. All the neighbouring regions are adjacent to each other with their extracted attributes. The relationship between these regions or vertices and their attributes is important for further processing.

The extracted features of the regions are represented as labels or attributes of the vertices or region in the RAG. After RAG formation each symbol or image is treated as a region adjacency graph with vertices as regions and edges as their inter-relationships. The perfection in feature extraction leads to more accuracy in the graph matching. The results of preprocessing the input image are shown in Figure 3. As discussed earlier, the preprocessing extracts constituent shapes of all the model symbols in the database. The status of model symbols after shape detection is listed in Table 1. It depicts the number of constituent basic shapes in each model symbol. Table 2 shows results of region separation and feature extraction for model symbols in the database. Overall, the database model symbols and the input image are described as the region adjacency graphs in this step.

Table 1. Model symbol database after shape detection.

\begin{tabular}{cccccccc}
\hline RAGid & Square & Rectangle & Triangle & Parallelogram & Quadrilateral & Rhombus & Trapezoid \\
\hline Bed & 0 & 1 & 2 & 0 & 0 & 0 & 0 \\
Pillar & 0 & 0 & 2 & 1 & 0 & 0 & 0 \\
Shelf & 0 & 0 & 4 & 0 & 0 & 0 & 0 \\
Staircase & 0 & 6 & 0 & 0 & 0 & 0 & 0 \\
Stove & 1 & 0 & 4 & 0 & 0 & 0 & 0 \\
Table & 0 & 9 & 0 & 0 & 0 & 0 & 0 \\
WC & 0 & 2 & 0 & 0 & 0 & 0 & 0 \\
Window & 1 & 3 & 0 & 0 & 0 & 0 & 0 \\
Door & 2 & 0 & 0 & 0 & 0 & 0 & 1 \\
TV & 0 & 2 & 0 & 0 & 0 & 0 & 2 \\
\hline
\end{tabular}



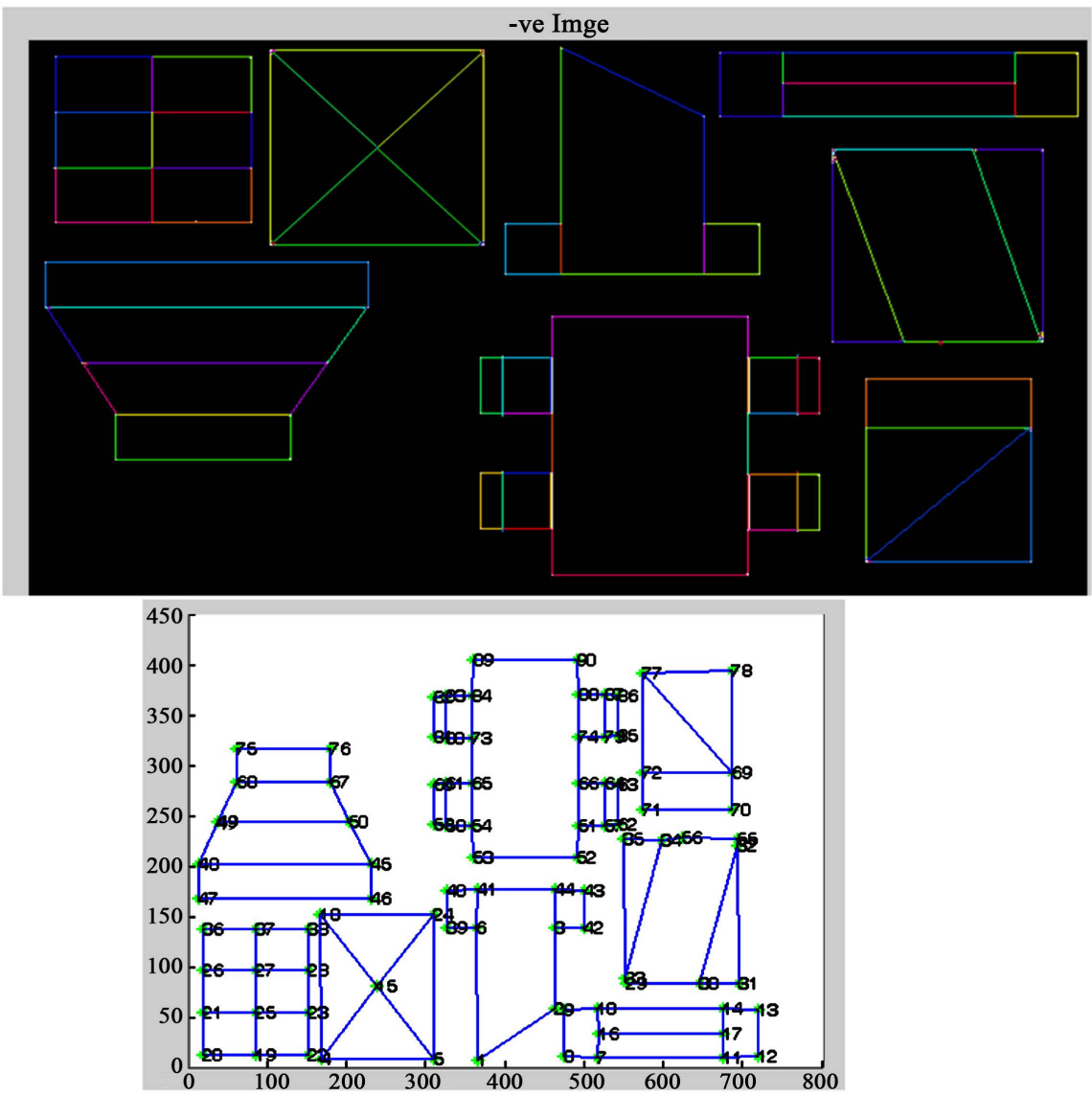

Figure 3. Result of input image preprocessing.

Table 2. Result of region separation and RAG construction.

\begin{tabular}{ccccc}
\hline RAGid & Nr of Region & Nr of Edges & Symbol Name & Priority \\
\hline Shelf & 4 & 4 & Shelf & 4 \\
Staircase & 6 & 7 & Staircase & 3 \\
Bed & 3 & 3 & Bed & 2 \\
Window & 4 & 5 & Window & 1 \\
Pillar & 3 & 2 & Pillar & 7 \\
Table & 9 & 8 & Table & 5 \\
Stove & 5 & 4 & Stove & 6 \\
TV & 4 & 3 & TV & 8 \\
Door & 3 & 2 & Door & 9 \\
\hline
\end{tabular}

The prototype symbols and the input image are both preprocessed and structural features like edges, junctions, corners, end points, closed regions, area, centroid, shape of region, neighborhood information of regions etc. are extracted from those images. Figure 3 shows negative of the input image and its corresponding structural details like edges, end points, corners, junctions identified during preprocessing. The closed regions are identified by edge tracing. The 
edge end points, junctions, corners are recorded as number of vertices for every closed region. While preprocessing, we also specify a tolerance value to extract these vertices for better a valuation and to show efficacy of our proposed method. The tolerance value greatly affects the result of preprocessing and so the result of symbol recognition as well. During the pre processing, shape of region is one of the important features useful for the database filtering. The status of model symbols database after shape detection is listed below in Table 1. It depicts the number of constituent basic shapes in each model symbol.

For example, Table 1 shows that, model symbol "bed" consists of one rectangle and two triangles as constituent shapes. The "pillar" consists of two triangles and one parallelogram; the "staircase" symbol consists of six rectangles as the constituent shapes. Table 2 shows results of region separation and some feature extraction for model symbols in the database. The features like "Number of regions", "Number of edges" are extracted for each of the model symbol. The "RAGid" is the identity number of the model symbol graph in the database. The "Priority" is the numeric value assigned to each symbol by the user for selecting a particular symbol to be matched against the input image. Table 1 and Table 2 are interlinked tables generated during feature extraction and preprocessing. In both tables, the first column consists of RAGid using model symbol name. Table 1 displays the primitive shapes detected in the respective model symbol depicted in first column. Table 2 depicts the detailed features like number regions, edges and priority in the model symbol. Here, a priority value represents in which order the model symbol is searched in the given input image. Lower the value of priority, that model symbol is searched first. Priority helps in linear sequencing of the matching process. The priority value for each model symbol is set by the user based on application which allows one to tune our proposed algorithm for a specific application.

\subsection{Graph Based Filtering}

After preprocessing, we apply graph based filtering algorithm which filters the database of model symbols with respect to the input image. It causes reduction in the number of symbols to be tested in the later phase of graph matching. It reduces the number of model symbols that are to be compared with the input image during graph matching. This filtering is done based on the constituent shapes of the input image and the constituent shapes of the model symbol graph database. The filtering algorithms are explained below.

\section{- Linear Filter}

In linear filtering, we identify the constituent shapes in the input image. The model symbol database is already equipped with all the features of all the model symbols during the preprocessing step of the database. In linear filtering the prototype symbol shapes and input image shapes are compared linearly. If the constituting symbol shapes match with each other then only further graph matching is performed. If the constituent shapes of both prototype graph and 
input graph do not match further graph matching is avoided. Figure 4 depicts the linear filtering.

As shown in Figure 4, consider two symbols $S_{1}$, a "staircase" and symbol $S_{2}$ as a "stove". The symbol $S_{n}$ is shown as a "bed". During the preprocessing, database records that $S_{1}$ comprises six rectangular shapes, $S_{2}$ constitutes four triangles and $S_{n}$ comprises one rectangle and two triangular shapes. Consider, "bed" is the input image. Preprocessing of input image identifies that the input image contains one rectangle and two triangular shapes. So, at the time of graph matching, $S_{1}$, $S_{2}, S_{\mathrm{n}}$, all three model symbols are compared with the input image as all three of them are containing either a rectangle or a triangle, which is there in the input image. If input image is a "staircase", preprocessing of input image concludes that input comprises six rectangular shapes. So the matching is performed with $S_{1}$ and $S_{n}$ only as they are having rectangle as one of their constituents. Comparison of the input with $S_{2}$ is avoided as $S_{2}$ do not have rectangle as its constituent. If input image is a "stove", comparison of input with $S_{1}$ is is avoided as $S_{1}$ do not have triangle as its constituent. Figure 4 elaborates this process.

\section{- Hierarchical Filter}

In hierarchical filtering the basic constituent shapes are arranged hierarchically in the database. The individual basic constituent shapes are at level 0 . At level 1, a combination of any two shapes is considered for filtering. At level 2 a combination of any 3 shapes is considered and so on the hierarchy goes on. The advantage of this type of hierarchy is that the shapes that do not occur in the

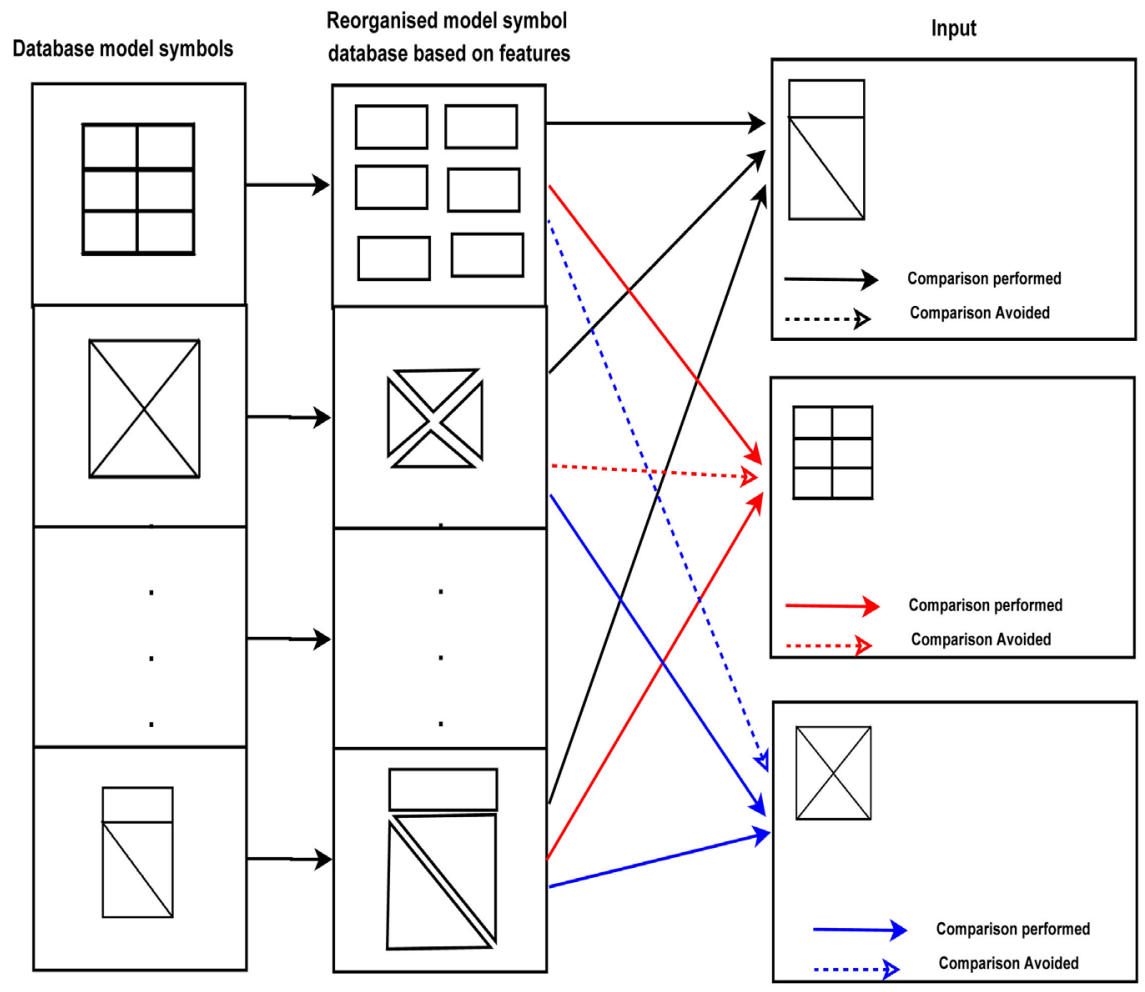

Figure 4. Linear filtering of the database. 
input image are skipped in graph matching algorithm, which further reduces high cost graph matching operations. Figure 5 and Figure 6 depict single level shape based representation of the model symbols. In Figure 5, the first column represents the database model symbol. The second column in Figure 5 represents the decomposed model symbols of respective database model symbol. After decomposition, the database is reorganized using indexing. The indexing is performed based on constituent shape. This step is illustrated using third and fourth column of Figure 5. The process of shape based indexing is further illustrated with an example in Figure 6, where the triangle and rectangle are primitive model shapes shown at the bottom in Figure 6 are indices to the database model symbols presented at the top in the figure. When an input image is presented for matching, the features of the input image are extracted. Based on the constituent shapes of the input image, shape based filtering is performed on the database which reduces the number of symbols to be matched. If the total database size is of say " $n$ " model symbols, only " $m$ " symbols are selected as relevant symbols to be matched with the input graph. The " $n$-m" models from the database are rejected as irrelevant symbols, and hence, " $n-m$ " symbols are not matched with the input image. The observation is that, shape detection and filtering procedure adds extra time to the database construction. But it is important to note that the database construction is a onetime offline activity. The decision, which symbol to be matched and which symbol not to be matched, is taken at the time of graph matching. In this context, our hierarchical filtering

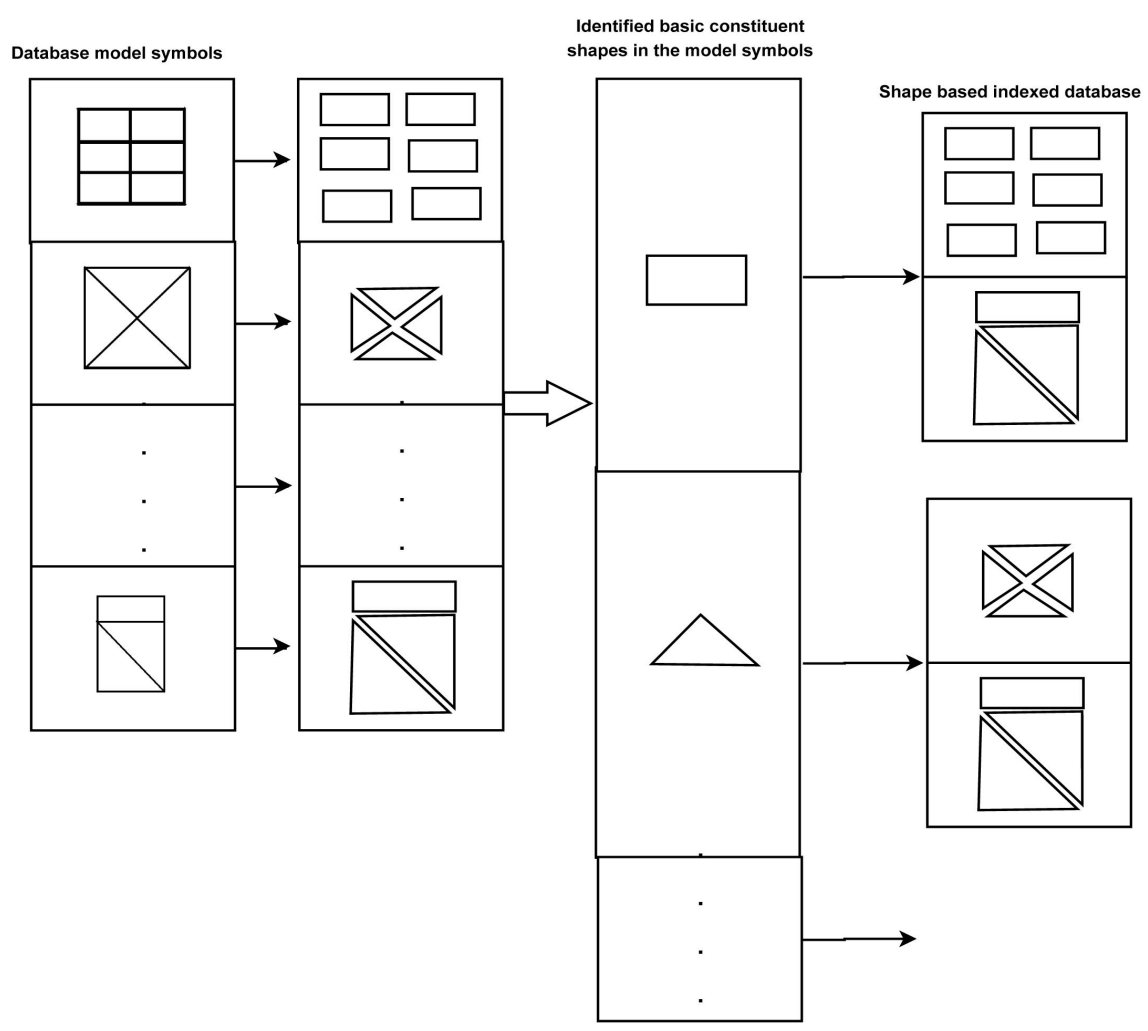

Figure 5. Hierarchical filtering of the database. 
Shape based indexing of the model symbols in the database
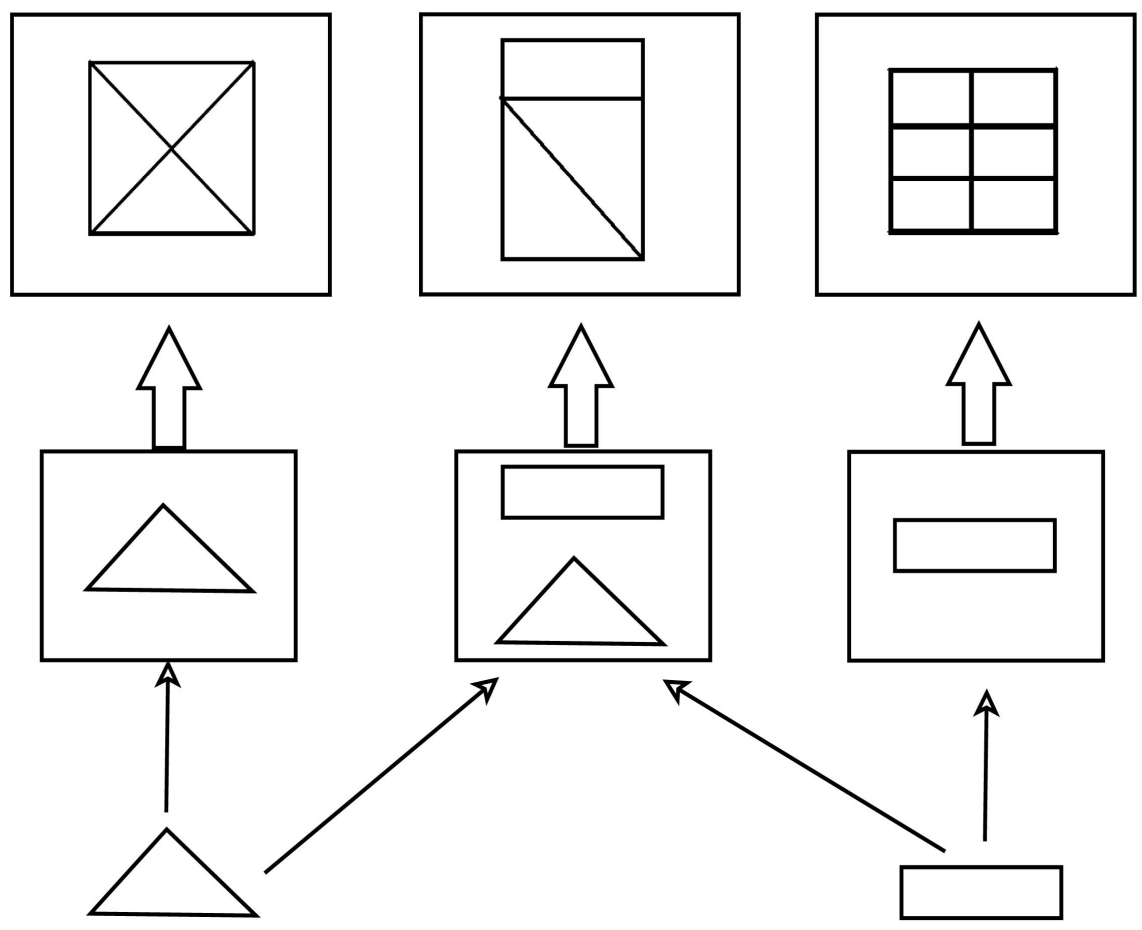

Figure 6. Hierarchical shape based indexing of the database.

approach provides an efficient solution for graph based matching. Few model symbols from the database are shown in Figure 7.

\subsection{Graph Matching Algorithm}

After the preprocessing and database construction, the graph based symbol matching is performed. For graph based matching, the RAG based string growing algorithm is applied for the architectural symbol recognition. An architectural plan containing various architectural symbols is given as an input. The identification of various model symbols from the database is performed in this step by using the string growing algorithm [7]. It reads each symbol one by one from the database and compares it with the input RAG. While comparing three basic operations are considered as substitute, shift and scale [7]. The substitution operation matches each region by comparing the regions. The shift operation matches the angular rotation to the neighbouring regions. The scaling cost matches expansion or shrinkage of the regions. The cost of mapping the input region with model region is calculated for each region. This cost is called as graph edit cost. The most promising match is accepted as a result. Figure 12 shows the results of symbol identification for different input images. The algorithm performs the following steps:

1) Read each prototype symbol linearly from the database.

2) For each closed input region perform polygon matching. Find substitution, shift and scaling cost. 


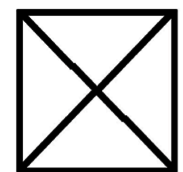

shelf

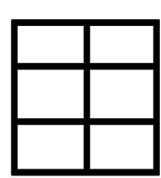

staircase

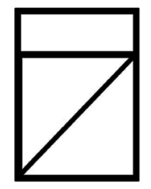

bed

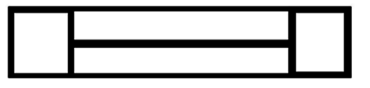

window

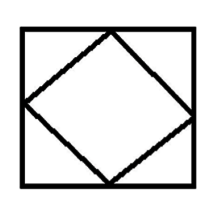

stove

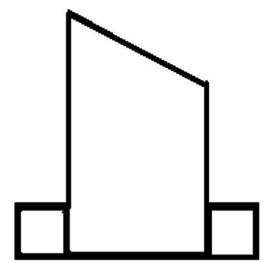

door

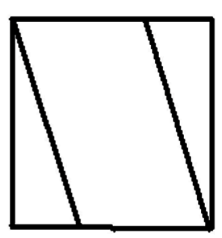

pillar

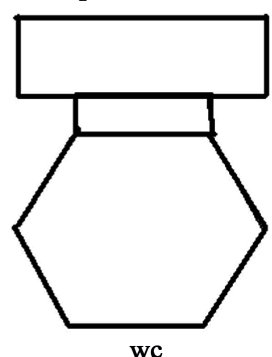

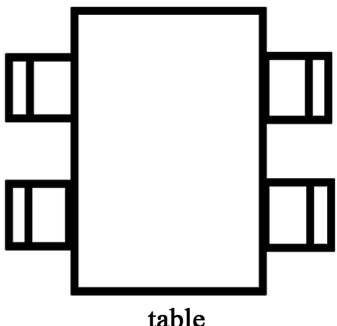

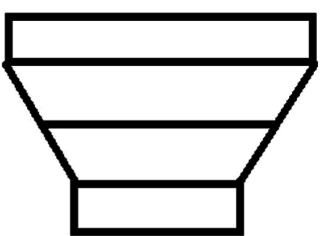

tv

Figure 7. Model symbol database.

3) Consider the regions, which are below threshold level for further matching.

4) Continue region matching till the prototype symbol is identified otherwise no match is found.

5) Conclude that the symbol is matching.

\section{Simulation Results}

We evaluated our proposed algorithm using architectural drawings. The input architectural plans are line drawing images. We consider both types of architectural images, 1) normal images, where all symbols are properly visible and 2) distorted images where the symbols are error-prone. For our implementation we have a model symbol database which constitutes the large number of model symbols. The few such architectural model symbols are depicted in Figure 7. We evaluate our proposed graph database filtering and matching algorithm using three different scenarios as below.

Scenariol 1: Symbol detection with graph matching without database filtering; Scenariol 2: symbol detection with linear filtering the graph database and Scenariol 3: symbol detection with hierarchical filtering of graph database.

During preprocessing and feature extraction, we introduce the tolerance value to determine accurate features of the image. Figure 8 elaborates role of tolerance value during preprocessing. Figure 8 shows an example with three vertices A, B and $\mathrm{C}$ identified during preprocessing of the image. For a stable value of tolerance, all the three vertices are detected. When we increase the tolerance, it looses vital vertex $B$ from the input. When we increase the tolerance, the number of vertices detected in the input image decreases. The decrease in number of 


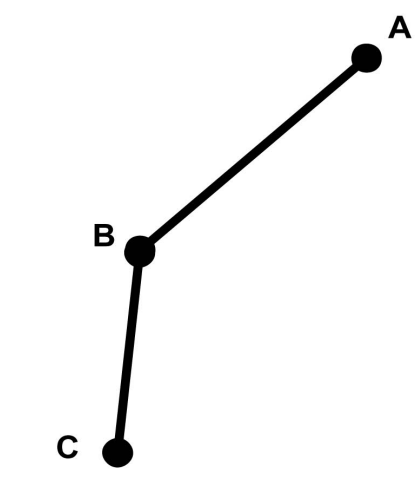

smaller tolerance value

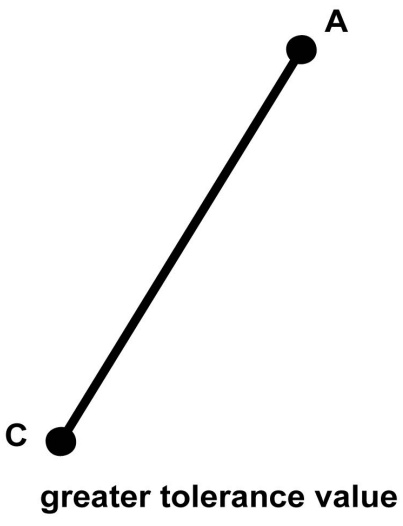

Figure 8. Vertex deletion due to tolerance.

vertices with increasing tolerance value is shown plotting the values in Figure 9. To a certain limit, the decrease in number of vertices is very sharp. The decreasing number of vertices signifies about loosing the features. For a specific value of the tolerance, number of vertices remains same. Figure 9 , shows the stable region of number of vertices from 72 to 69 , where tolerance value is ranging from 5 to 19. After this stability, the further increase in the tolerance results in loss of vertices which are at vital position in the image. Deletion of these vertices changes the basic shape of a symbol in the input image. This is the reason preprocessing affects the global results of symbol detection.

Figure 10, shows the effect of tolerance on the symbol recognition. When tolerance value is 8 , number of vertices identified in the feature extraction is 72 . When tolerance is 10 , number of vertices is 71 . It looses the significant vertex number 18 , which is critical for identifying the symbol door in the image. So the resultant output of graph matching fails to identify symbol door as shown in Figure 10 with tolerance value 10 .

Figure 11, shows the graph of tolerance in relation with number of vertices identified during feature extraction and the number of symbols identified. The tolerance value is varied from 1 to 5 . The output shows that the system remains stable and identifies maximum number of symbols when tolerance value remains 2 to 5. Ideally, we keep tolerance value 3 or 4 for further testing.

After the preprocessing, the model symbol database is reorganized in hierarchical manner based on the constituent shapes. The model symbols are indexed on the basis of constituent shapes of the models. When an input image is given, the graph isomorphism algorithm is applied to recognize model symbols in it. Figure 12 shows identified symbols in the input image. Various symbols "staircase", "door", "table", "window", "shelf" are identified with variable error tolerance for the Scenario 1.

Figure 13, shows the result of symbol recognition in the hand drawn image. Its important to observe that the algorithm works equally good with the hand drawn images which are not very clear and noisy too. Various symbols like staircase, windows, table, shelf, and door are identified in the input image. 


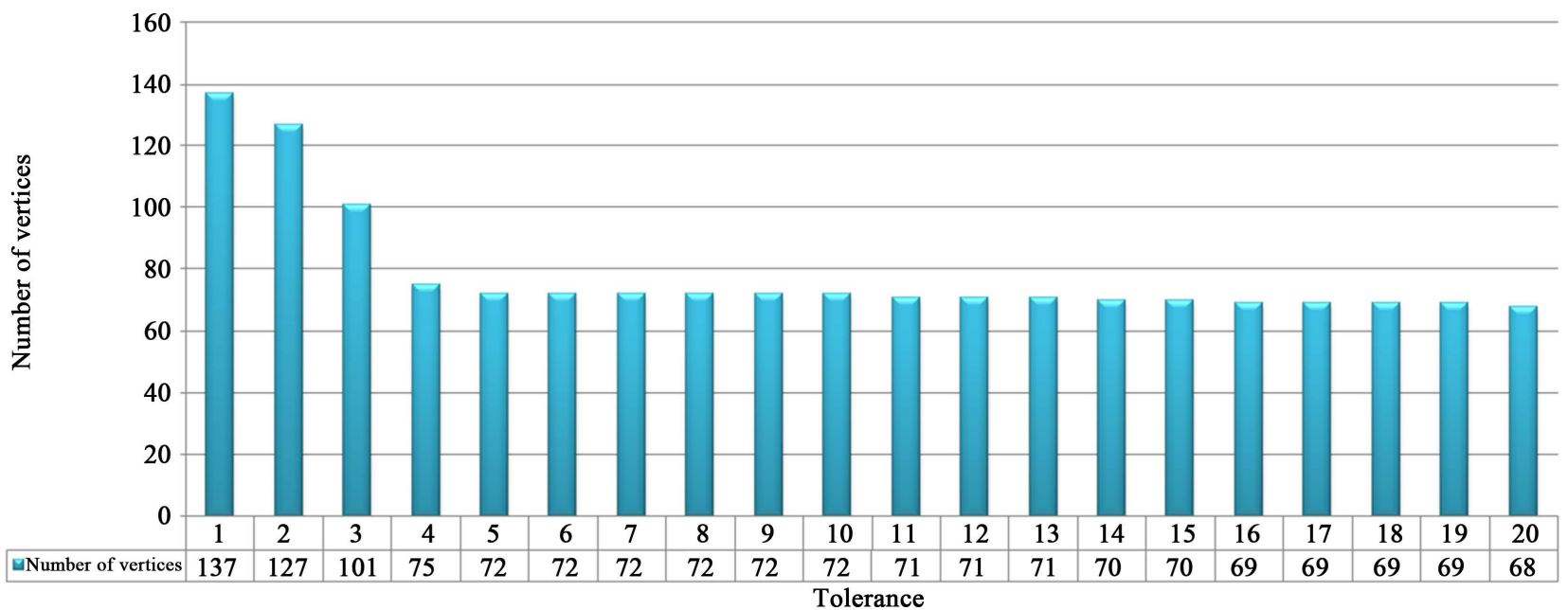

Figure 9. Effect of tolerance on vertices.

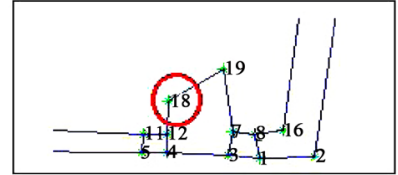

Tolerance 8

Vertices 72

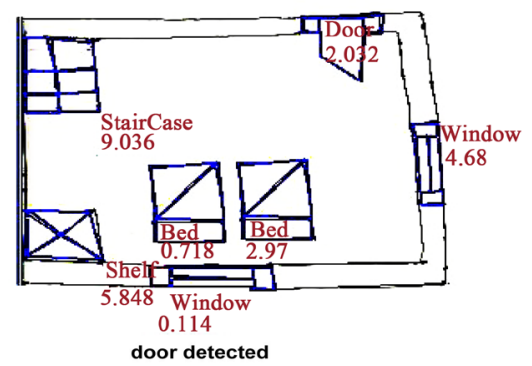

Figure 10. Tolerance and symbol recognition.

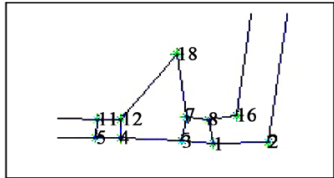

Tolerance 10

Vertices 71

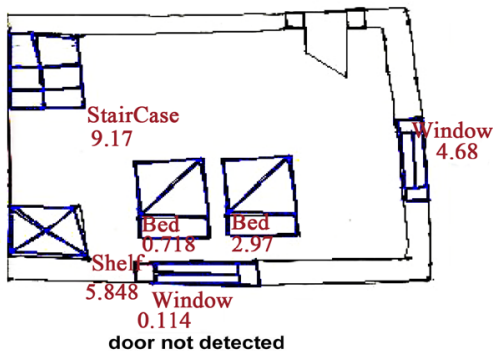

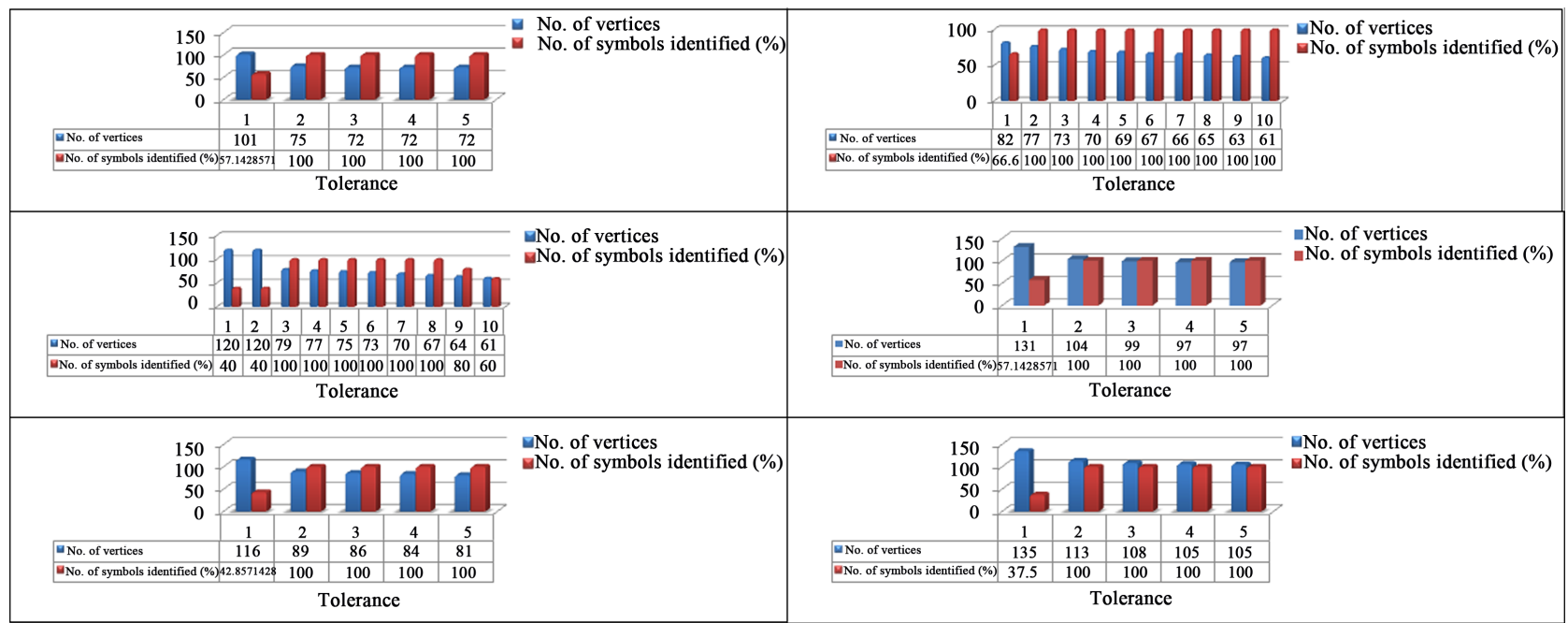

Figure 11. Effect of tolerance on performance. 


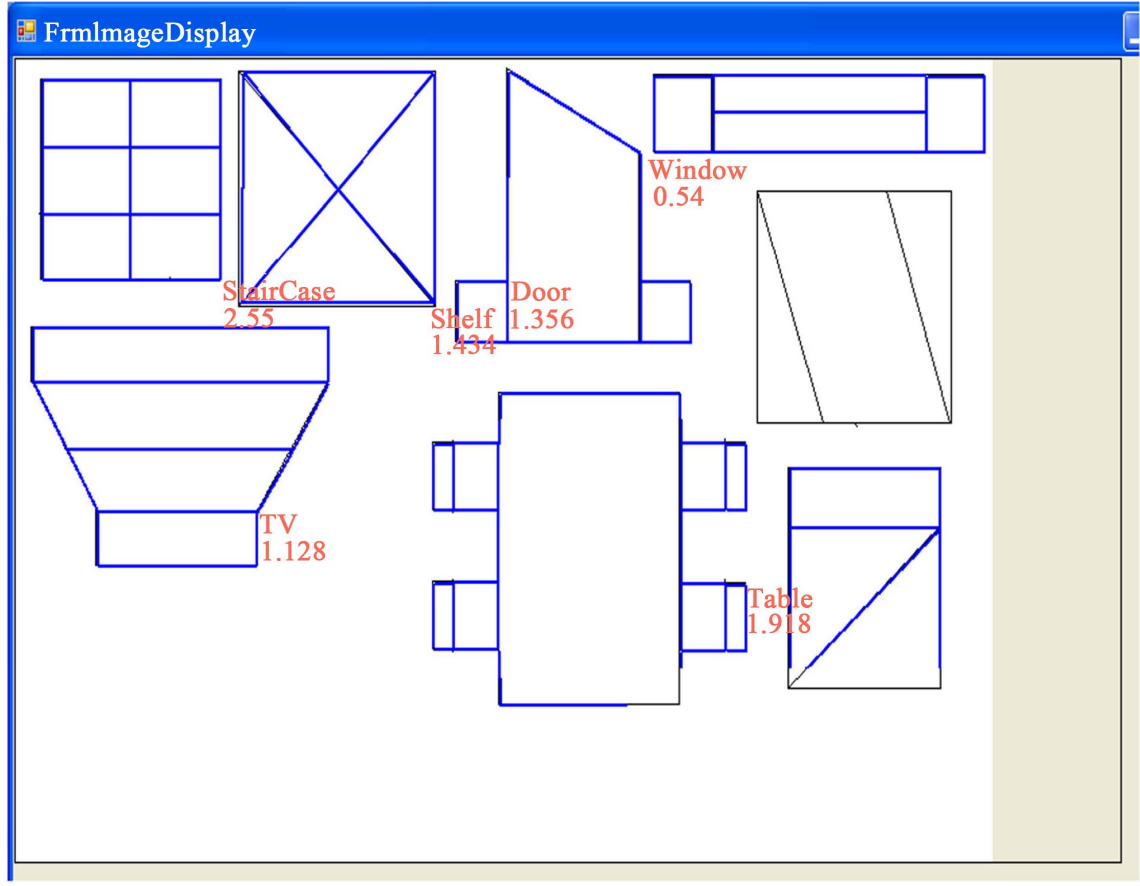

Figure 12. Symbol detection output.

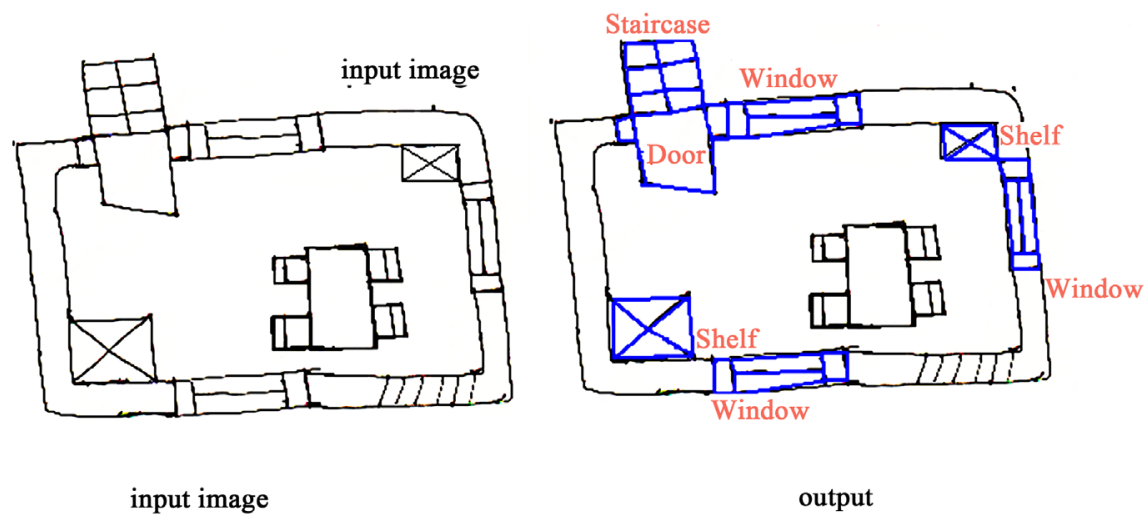

Figure 13. Symbol detection in noisy image.

Figure 14 depicts another example of symbol recognition with hand drawn image. It is observed that if the image contains both very small and very large symbols at same time then the error tolerance factor given for graph matching fails to balance. The error tolerance introduced before graph matching, cannot suit to find very small and very large symbols at the same time. This is because, the smaller symbols need lower tolerance value for accurate features extraction where as the larger symbols can be identified with higher tolerance values. For a stable system, the input image must contain average size symbols.

We observe a considerable variation in string edit cost [7] for each symbol for the variation in the error tolerance. After a attaining a certain tolerance value, the edit cost, the number of symbols identified in image and the number of vertices considered in an input image after preprocessing remain constant and the 


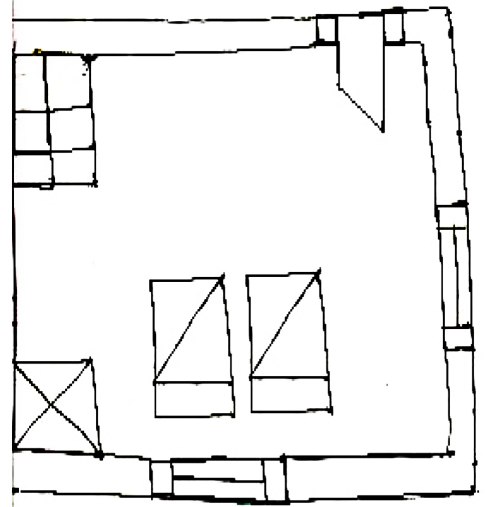

input image

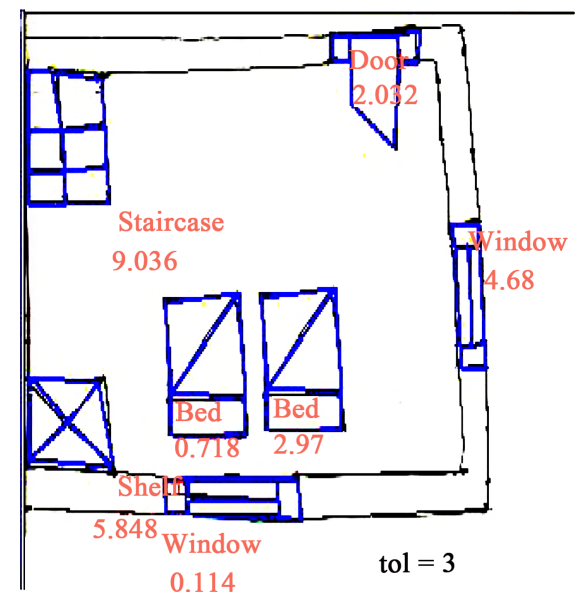

output

Figure 14. Symbol recognition in hand drawn image.

system gets a particular level of stability for that tolerance limit. The error tolerance value is subjective to accuracy expected by the various applications. There cannot be any automatic inference method for tolerance which can suit globally to all pattern matching applications. Its estimation is a challenging job which purely depends on the type of application.

Figure 15 shows that the graph matching algorithm shows robustness to distortion, rotation, scaling and rotation. If the input image is distorted in such a way that it is not possible to extract a primitive graph, the decomposition will not be proper. In such a case we need additional pre-processing step so that the smallest closed region can be extracted as a primitive graph and can be used as a sub graph for the complete representation of an input drawing in terms of graph. It is also important to note that the simple shape based comparison will not work as the shapes are not predefined and an input drawing may not have exact primitive shapes only. There may be variety of shapes which may be difficult to represent in terms of primitive shapes. In such case, the direct shape based comparison is not feasible. Moreover, this increases the computational complexity heavily compared to RAG based analysis and decomposition of an input drawing into sub graph based representation. We have demonstrated the efficacy of our proposed symbol recognition using input drawings where the images are not clearer. Only point is that if the input image is too much distorted definitely we need to pre-process the input image for better extraction of primitive region which forms primitive sub graph. The graph based representation can tolerate errors and distortions in the graph matching as exact shape matching is not performed. We have applied the shape detection and filtering algorithm to the model symbol database. The number of comparisons done and number of comparisons avoided are plotted in Figure 16 for the linear filtering. While plotting the graph, the image samples on the $\mathrm{x}$-axis are arranged in the increasing order of the size of the input image. The images containing more 
number of symbols take more number of comparisons. The conclusion is that on an average 2/3 number of times comparisons are avoided due to filtering than that of without filtering which is significant achievement.

There is an acceleration gain using hierarchical graph filtering. By inclusion of the filtering stage overall efficiency is enhanced for graph based symbol recognition compared to graph matching without filtering.

We compared the time taken for graph processing without using filtering [7]
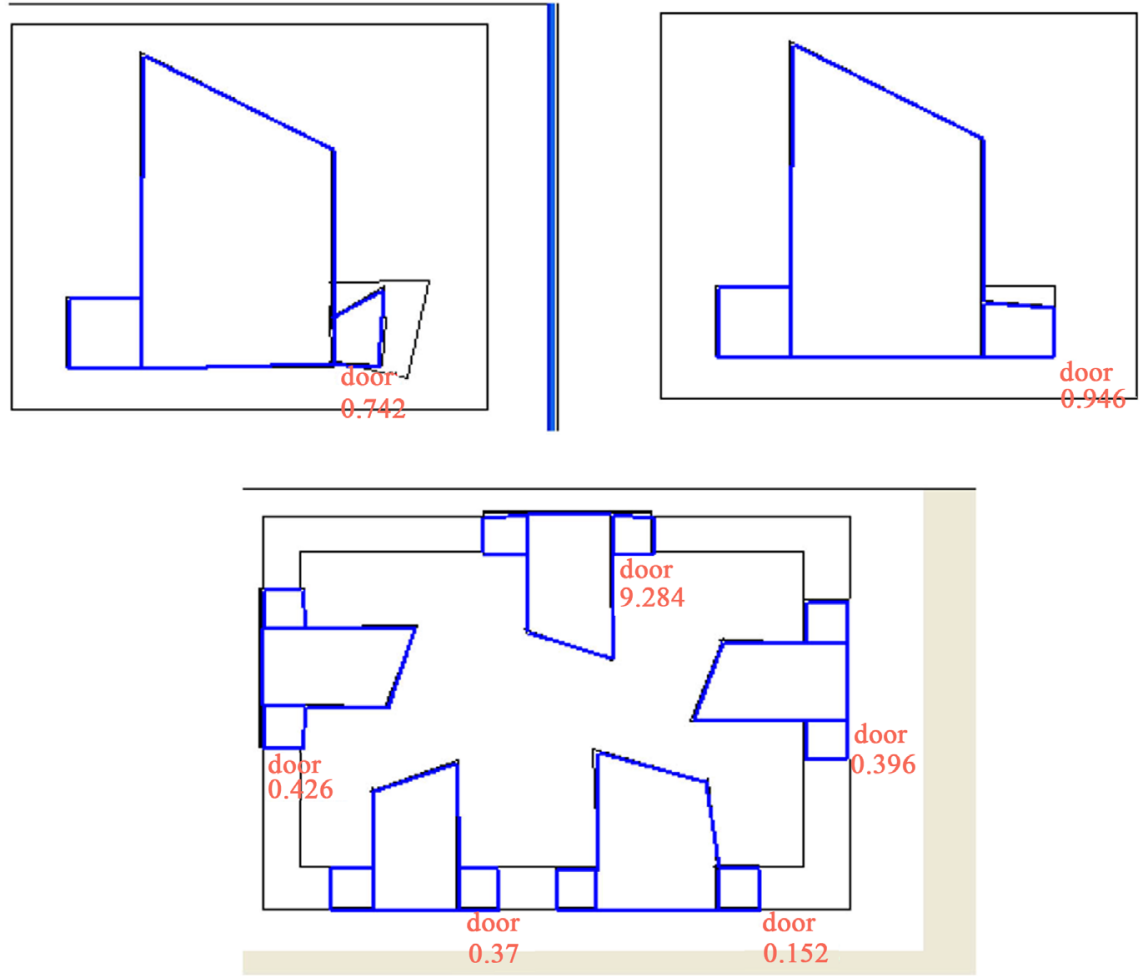

Figure 15. Distorted symbol recognition.

Linear filtering

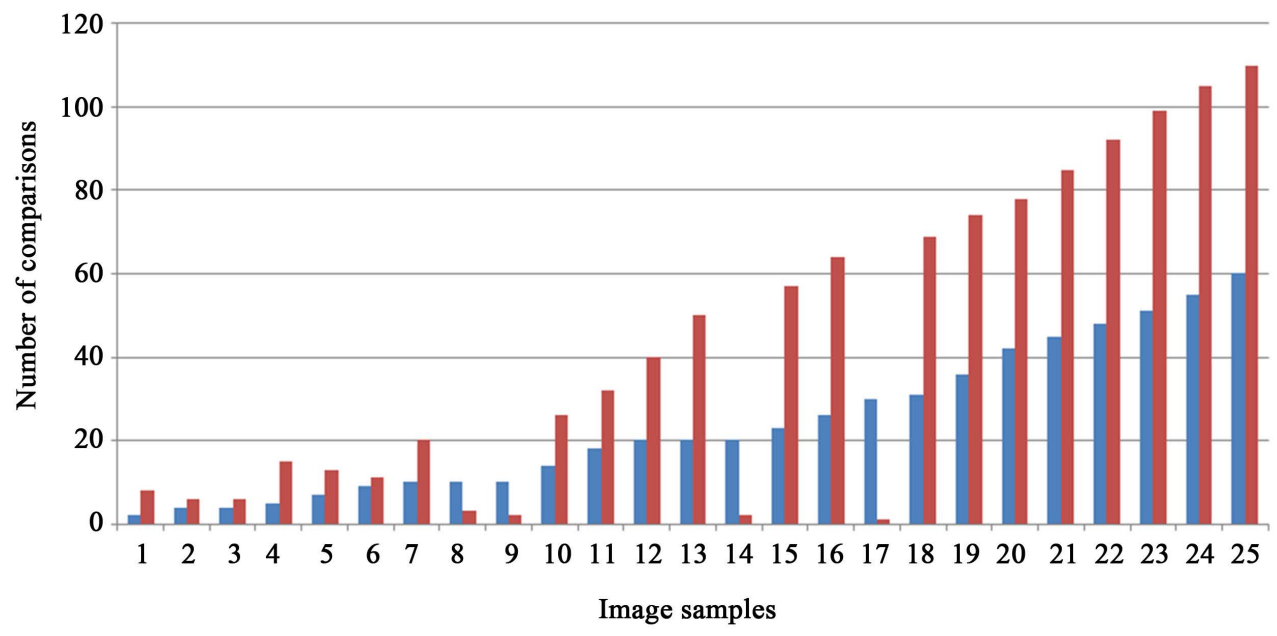

Comparisons done

Comparisons avoided

Figure 16. Number of comparisons using linear filtering. 
and with filtering. Figure 17 shows the results of time comparison for graph processing time with graph filtering algorithm and without graph filtering algorithm. The images on the $\mathrm{x}$-axis are arranged in the increasing order of their sizes. The first image is the smallest image with minimum number of regions while the last image sample shows the largest image with maximum number of regions. When we do not apply filtering, the graph processing time is minimum as shown in the graph 17. The linear filtering takes little more time than that of one without filtering.

The hierarchical filtering algorithm takes maximum graph processing time in all the three scenarios. We compared the time taken for graph matching without using filtering [7] and with filtering. Figure 18 shows the comparison of graph

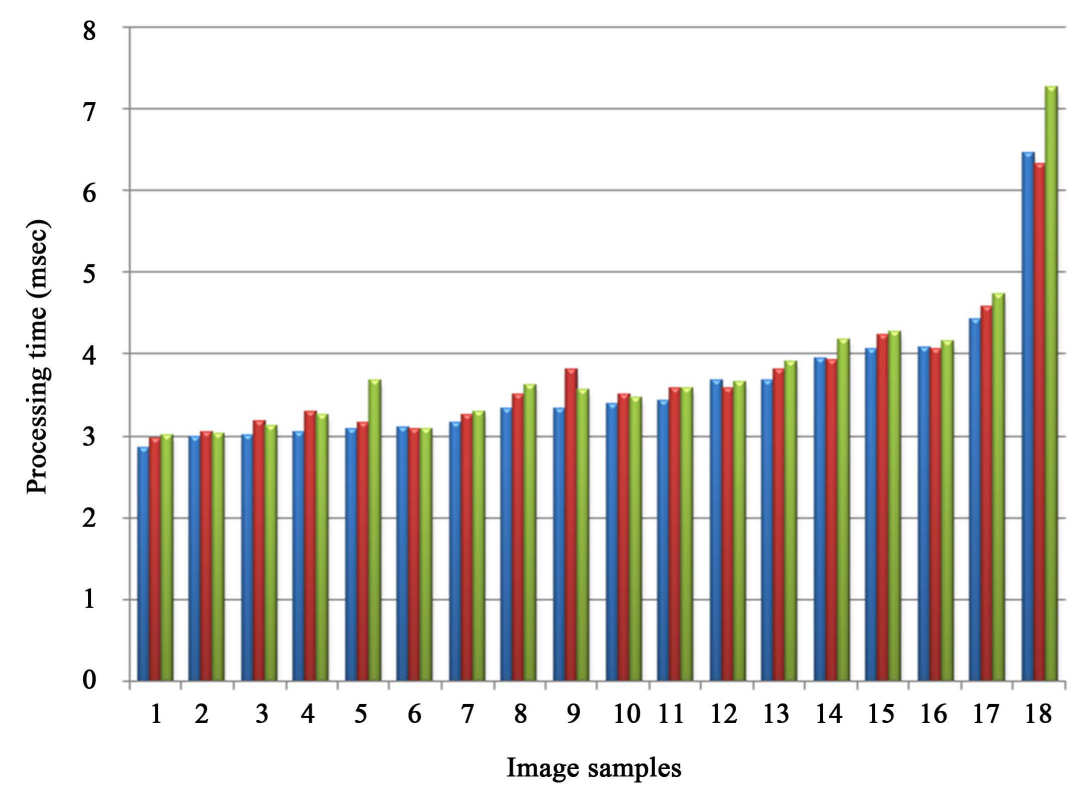

- Matching without filtering [29]

- Linear filtering

$\square$ Hierarchical filtering

Figure 17. Graph processing time using filtering.

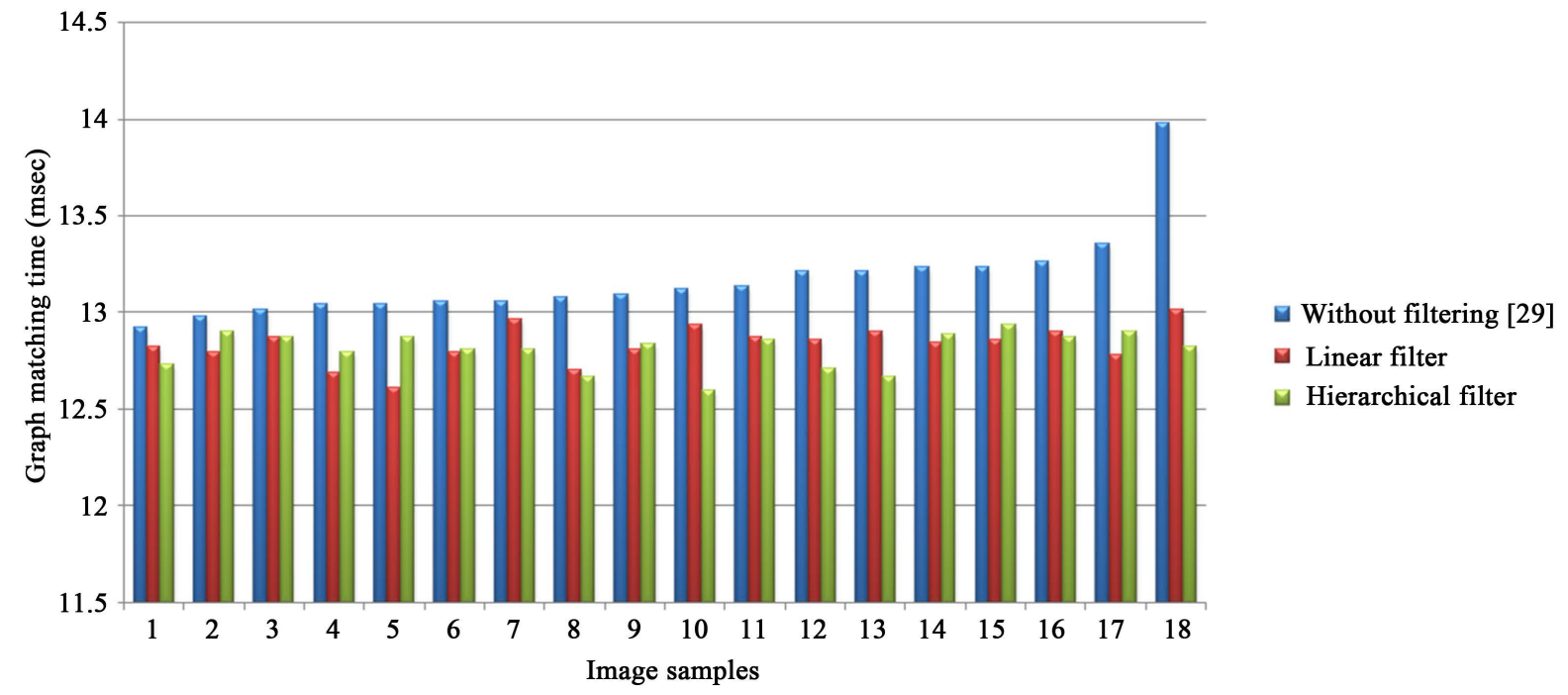

Figure 18. Graph matching time using filtering. 
matching time taken for symbol recognition with graph filtering and without graph filtering [7]. It is clearly seen that the graph matching time without filtering for all the samples is maximum. In eight of the samples out of eighteen, linear filtering is performing better than hierarchical filtering time. In other ten samples out of eighteen images, hierarchical filter based graph matching time is the minimum one. We evaluated the proposed method with large number of input drawings of different varieties which include basic symbols, hand drawn images, distorted images and architectural plans. The thumbnails of images we have used for testing the algorithm are shown in Figure 19. The important point is that some of input drawings have 40 closed regions. In the simulation results we have depicted some of them.

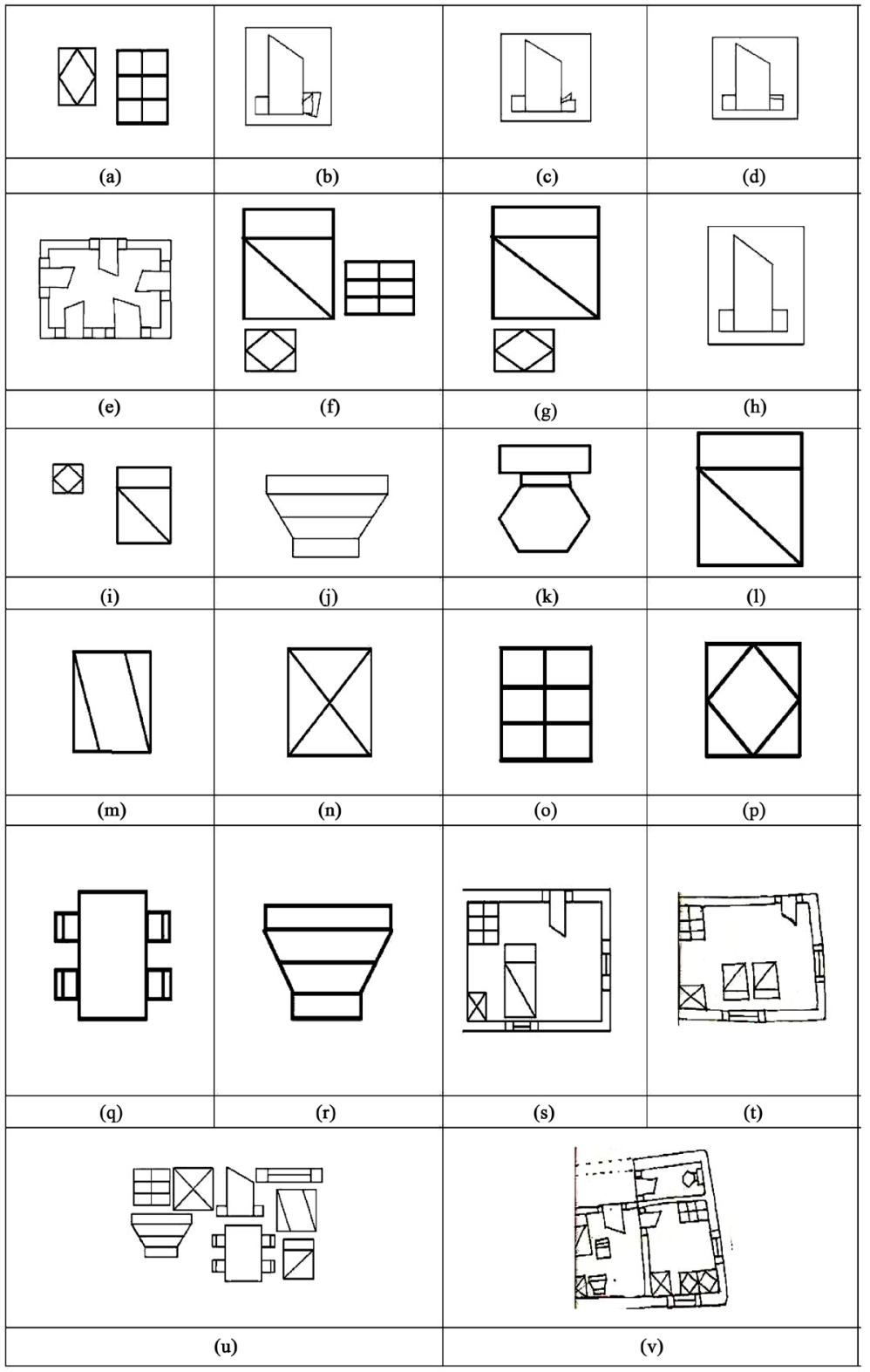

Figure 19. Thumbnail images used for testing. 


\section{Conclusion}

In this work we have proposed and evaluated graph filtering and matching for symbol recognition. Architectural plan images are provided as input to the algorithm. The graph matching is done by region adjacency graph matching [7] [8] based on string growing matching. It is observed that when we use only graph matching (without filtering) the number of comparisons done is more as compared with graph matching with filtering. Filtering prior to matching reduces drastically the number of matching model symbols available in the database averagely by $2 / 3$ times. Ultimately this reduces the time taken for pure matching process, although the graph processing time increases more rapidly with symbol recognition with graph filtering than that of without filtering. In that case it is expected that graph databases processing and filtering is to be done offline prior to graph matching process for best results as a combination. The algorithm for graph matching has very strong restrictions about images due to "specificness" of symbols for different applications. It's always a challenge to achieve a general platform for various applications and their distortion accepting capacities and needs.

\section{Conflicts of Interest}

The authors declare no conflicts of interest regarding the publication of this paper.

\section{References}

[1] Ullman, J.R. (1976) An Algorithm for Subgraph Isomorphism, Journal of ACM, 23, 31-42. https://doi.org/10.1145/321921.321925

[2] Fernandez, M.L. and Valiente, G. (2001) A Graph Distance Metric Combining Maximum Common Subgraph and Minimum Common Supergraph. Pattern Recognition Letters, 22, 753-758. https://doi.org/10.1016/S0167-8655(01)00017-4

[3] Raymond, J.W. and Willett, P. (2002) Maximum Common Subgraph Isomorphism Algorithms for the Matching of Chemical Structures. Journal of Computer-Aided Molecular Design, 16, 521-533. https://doi.org/10.1023/A:1021271615909

[4] Messmer, B. and Bunke, H. (1998) A New Algorithm for Error-Tolerant Subgraph Isomorphism Detection. IEEE Transactions on Pattern Analysis and Machine Intelligence, 20, 493-505. https://doi.org/10.1109/34.682179

[5] Conte, D., Foggia, P., Sansone, C. and Vento, M. (2004) Thirty Years of Graph Matching in Pattern Recognition. International Journal of Pattern Recognition and Artificial Intelligence, 18, 265-298. https://doi.org/10.1142/S0218001404003228

[6] Cordella, L.P., Foggia, P., Sansone, C. and Vento, M. (2004) Subgraph Isomorphism Algorithm for Matching Large Graphs. IEEE Transactions on Pattern Analysis and Machine Intelligence, 26, 1367-1372.

[7] Llados, J., Marti, E. and Villanueva, J.J. (2001) Symbol Recognition by Error-Tolerant Subgraph Matching between Region Adjacency Graphs. IEEE Transactions on Pattern Analysis and Machine Intelligence, 23, 1137-1143. https://doi.org/10.1109/34.954603

[8] Sanchez, G., Llados, J. and Tombre, K. (2002) An Error Correction Graph Grammar 
to Recognize Textured Symbols. In: Blostein, D. and Kwon, Y.B., Eds., Graphics Recognition Algorithms and Applications, Springer, Berlin, Heidelberg, 128-138.

[9] Garey, M.R. and Johnson, D.S. (1979) Computers and Intractability: A Guide to the Theory of NP-Completeness. W. H. Freeman \& Co., New York.

[10] Cook, S.A. (1971) The Complexity of Theorem-Proving Procedures. Proceeding of 3rd ACM Symposium on Theory of Computing, Shaker Heights, 3-5 May 1971, 151-158. https://doi.org/10.1145/800157.805047

[11] Luks, E.M. (1980) Isomorphism of Graphs of Bounded Valence Can Be Tested in Polynomial Time. 21 st Annual Symposium on Foundations of Computer Science, Syracuse, 13-15 October 1980, 42-49.

[12] Datta, S., Limaye, N., Nimbhorkar, P., Thierauf, T. and Wagner, F. (2009) Planar Graph Isomorphism Is in Log-Space. 24th Annual IEEE Conference on Computational Complexity, Paris, 15-18 July 2009, 203-214. https://doi.org/10.1109/CCC.2009.16

[13] Foggia, P., Sansone, C. and Vento, M. (2001) A Performance Comparison of Five Algorithms for Graph Isomorphism. Proceeding of the $3 \mathrm{rd}$ IAPR Workshop on Graph Based Representations in Pattern Recognition, 23 May 2001, 188-199,

[14] Bunke, H. (1999) Error Correcting Graph Matching: On the Influence of the Underlying Cost Function. IEEE Transactions on Pattern Analysis and Machine Learning, 21, 917-922.

[15] Joyner, I. and Holder, L.B. (2001) Graph-Based Hierarchical Conceptual Clustering. Journal of Machine Learning Research, 2, 19-43.

[16] Irniger, C. and Bunke, H. (2005) Decision Trees for Error-Tolerant Graph Database Filtering. 5th IAPR International Workshop, Vol. 3434, Poitiers, 11-13 April 2005, 301-311.

[17] Sengupta, K. and Boyer, K. (1995) Organizing Large Structural Model Bases. IEEE Transactions on Pattern Analysis and Machine Intelligence, 17, 321-332. https://doi.org/10.1109/34.385984

[18] Shapiro, L. and Haralick, R. (1982) Organization of Relational Models for Scene Analysis. IEEE Transactions on Pattern Analysis and Machine Intelligence, 3, 595-602. https://doi.org/10.1109/TPAMI.1982.4767312

[19] Sossa, H. and Horaud, R. (1992) Model Indexing: The Graph-Hashing Approach. Proceedings of IEEE Conference on Computer Vision and Pattern Recognition, Champaign, 15-18 June 1992, 811-814.

[20] Messmer, B. and Bunke, H. (1999) A Decision Tree Approach to Graph and Subgraph Isomorphism. Pattern Recognition, 32, 1979-1998. https://doi.org/10.1016/S0031-3203(98)90142-X

[21] Riesen, K. and Bunke, H. (2009) Dissimilarity Based Vector Space Embedding of Graphs Using Prototype Reduction Schemes. Proceedings of the 6 th International Conference on Machine Learning and Data Mining in Pattern Recognition, Leipzig, 23-25 July 2009, 617-631.

[22] Irniger, C. and Bunke, H. (2003) Theoretical Analysis and Experimental Comparison of Graph Matching Algorithms for Database Filtering. Proceedings of 4 th IAPR International Workshop on Graph Based Representations in Pattern Recognition, Vol. 2726, 30 June-2 July 2003, 118-129. https://doi.org/10.1007/3-540-45028-9_11

[23] Legaspe, E.P., Silva, W.S., Fontana, C.F. and Dias, E.M. (2011) Automatic Character Recognition Based on Graph Theory: A New Approach to Automation. The Pro- 
ceedings of the 2nd International Conference on Circuits, Systems, Communications and Computers, Puerto De La Cruz, 71-79.

[24] Li, M.J. and Dai, R.W. (1995) A Personal Handwritten Chinese Character Recognition Algorithm Based on the Generalized Hough Transform. The Proceedings of the 3 rd International Conference on Document Analysis and Recognition, Vol. 2, Montreal, 14-16 August 1995, 828-831.

[25] Gao, X., Xiao, B., Tao, D. and Li, X. (2010) A Survey of Graph Edit Distance. Pattern Analysis and Applications, 13, 113-129. 